\title{
Valentin Boyer
}

Paris

ENTRE RÊVERIE ET AUTHENTICITÉ - UNE IMMERSION DANS L’ÉGYPTE ANCIENNE AVEC LE PEINTRE STEFAN BAKAŁOWICZ ${ }^{1}$

\begin{abstract}
This article consists of a study of two Egyptian paintings of the Russian-Polish painter Stefan Bakalowicz (1857-1947). The study seeks to establish the degree of authenticity and credibility of the represented patterns by finding the sources of archaeological inspirationboth in European Egyptian collections and in the Egyptological works used by artists in the 19th century-which inspired (or could potentially inspire) Bakalowicz to carry out the staging and reconstruction of ancient Egypt. Furthermore, the study focuses on Bakalowicz's artistic approach and bias as a representative of the late Academicism at the turn of the 20th century. This research is based on the study of the arrangement, choice, and evocative scope of particular patterns as well as the role of fantasy in the service of a theatrical staging of the past Egypt. It also aims to discern elements relating to Egyptomania, Orientalism or even pure academism.

Keywords: Egyptomania; Bakalowicz; Ancient Egypt; Orientalism; Academism, Painting; Polish Artist; Egyptian Revival; Art History; Reception of Ancient Egypt; Archaeology

\footnotetext{
1 L'article a été rédigé sur la base du mémoire de recherche non publié : Boyer V. 2019. Die ägyptisierenden Gemälde des russisch-polnischen Malers Stefan Bakałowicz (1857-1947). Zwischen Ägyptomanie und Ägyptophilie: Inspirationen, Einflüsse sowie künstlerische und ästhetische Ansätze eines Malers des Spätakademismus. Unpublished MA dissertation, University of Heidelberg. Heidelberg.
} 
[L'égyptomanie] continue de se développer malgré les parutions successives à travers l'Europe, tout au long du siècle, d'ouvrages de mieux en mieux documentés et illustrés, parmi lesquels ceux de Champollion, Ippolito Rosellini, Auguste Mariette, Karl Lepsius et Émile Prisse d'Avennes. Ceux-ci sont à leur tour rapidement détournés et leurs gravures servent aux artistes de sources d'inspiration renouvelées. Néanmoins, il ne faudrait pas croire que l'égyptomanie s'est construite uniquement sur de tels modèles livresques. Les artistes réinterprètent volontiers la réalité pour inventer des formes égyptisantes audacieuses qui deviennent à leur tour de nouvelles sources d'inspiration (Hennebert 2012, 47).

L'admiration et la fascination de l'Égypte ne sont-elles pas les points de départ du désir de reproduire un environnement antique avec du mobilier et des artefacts décoratifs contextualisants pour représenter une Égypte ancienne éternelle et immuable?

Établir le degré d'authenticité et de crédibilité des motifs représentés en retrouvant les sources d'inspirations archéologiques - dans les collections égyptiennes ou dans les ouvrages égyptologiques utilisés par les artistes au XIX ${ }^{\text {ème }}$ siècle - qui ont pu inspirer Bakałowicz dans ses représentations de l'Égypte ancienne, permet de discerner la part de fantaisie et de rêverie. Toutefois, toute représentation de l'Égypte ancienne ne doit pas nécessairement être considérée uniquement par le prisme de la tradition égyptomaniaque mais également de celui de l'orientalisme.

Né à Varsovie le 10 octobre 1857 et décédé à Rome le 02 mai 1947 à l'âge de 89 ans, fils de Władislaw Bakałowicz et de Wiktoryna Szymanowska et marié à l'italienne Giuseppina Aloisi ${ }^{2}$, Stefan Bakałowicz ${ }^{3}$ (1857-1947) est un peintre d'histoire russo-polonais ${ }^{4}$, représentant de l'académisme tardif au tournant du $\mathrm{XX}^{\text {ème }}$ siècle, période durant laquelle le contexte artistique était en pleine mutation. Étudiant à l'Académie impériale de Saint-Pétersbourg de 1876 à 1881 et après avoir accumulé de nombreuses

\footnotetext{
2 Informations présentes dans l'Acte de décès de Stefan Bakałowicz et dans une note manuscrite biographique (Tous mes remerciements à Monsieur Michel Bakałowicz, petitfils du demi-frère de Stefan Bakałowic, de m'avoir transmis ces documents).

3 En russe : Бакалович, Степан Владиславович, transcription : Bakalovič, Stepan Vladislavovič.

4 Bakałowicz était polonais mais sujet russe. Dans la seconde moitié du XIX ${ }^{\text {ème }}$ siècle, la Pologne n'existe pas comme entité nationale, et ce jusqu'à son indépendance en novembre 1918, reconnue par le petit traité de Versailles en juin 1919. Elle est partagée entre l'Empire russe, la Prusse (puis l'Allemagne) et l'Autriche-Hongrie.
} 
médailles académiques, Bakałowicz en devient pensionnaire de 1881 à 1885 (Benezit, 321) obtenant de l'Académie de Saint-Pétersbourg une bourse de voyage pour une durée de cinq ans (Casali et Caron-Lanfranc de Panthou 2011, 140) dont il profitera pour se rendre tout d'abord à Cracovie, puis à Paris, en Algérie, en Lybie et enfin à Rome. En 1881, il entame donc son voyage à l'étranger de cinq ans par un retour en Pologne à Cracovie ${ }^{5}$ afin d'y travailler à l'Ecole des Beaux-Arts auprès du peintre polonais Jan Matejko (1838-1893) (Богословская И. 2016, 390) avec lequel il réalise le célèbre tableau historique « Bataille de Grunwald ${ }^{6}$ qui en fait le plus grand peintre historique polonais ${ }^{7}$. Cependant, il est à noter que Jan Matejko a préféré faire une synthèse historique dans ses peintures à la gloire de l'histoire polonaise plutôt que de s'attacher à la véracité historique, notamment en représentant de grands personnages n'ayant pas forcément participé aux faits historiques dépeints. Cet imaginaire historique polonais ne sera pas le credo de Bakałowicz, qui toutefois, s'attachera quant à lui à représenter dans nombre de ses peintures aussi bien la Rome, la Grèce que l'Égypte antiques, en faisant preuve d'une certaine imagination nourrie par de minutieuses représentations archéologiques. Son œuvre compte ainsi plusieurs tableaux égyptisants de grande qualité, encore inédits à ce jour. Au cours de sa carrière, il aura ainsi voyagé en Pologne, en Russie, en France, en Italie, en Angleterre, en Algérie, en Libye, en Tunisie ainsi qu'en Égypte. Stefan Bakałowicz s'est rendu en Égypte seulement en $1903^{8}$ (A. D. 1906, 642643 ) et a introduit des thèmes orientaux dans ses peintures après ce voyage (Derwojed 1971, 72), bien qu'il ait déjà réalisé deux toiles égyptisantes avant de s'y rendre ${ }^{9}$ : «La danse égyptienne » en 1888 ainsi que « Le scribe

5 Depuis 1866, la province galicienne de Cracovie bénéficie d'un statut autonome en contrepartie de sa loyauté à l'Autriche-Hongrie, lui permettant de devenir un centre culturel et artistique. Cracovie attire désormais de nombreux peintres, écrivains et poètes comme Jan Matejko.

${ }^{6}$ Jan Matejko, Bataille de Grunwald, 1878, 426 x 987cm, huile sur toile, Musée national de Varsovie.

7 Golitsyn I. Bakalovich, Stepan Vladislavovich. In The Rodon Foundation. Extrait de http://www.rodon.org/art-071206093316 [consulté le 15.08.2020]. Tous mes remerciements à Madame Aneta Biały, conservatrice adjointe de la collection d'art polonais d'avant 1914 du musée national de Varsovie, pour la traduction du texte russe en anglais.

${ }^{8}$ En 1904 d'après la revue polonaise Tygodnik Illustrowany. Tous mes remerciements à Madame Aneta Biały, conservatrice adjointe de la collection d'art polonais d'avant 1914 du musée national de Varsovie, pour la traduction du texte polonais en anglais.

9 Il est à noter qu'il n'existe pas de différence notable entre les tableaux réalisés avant et après son voyage en Egypte en 1903. «Le scribe royal Rahotep » réalisé en 1901, soit avant son voyage, pourrait laisser penser que Bakalowicz s'attachait moins à la représentation 
royal Rahotep » en 1901. D'après la revue polonaise Tygodnik Illustrowany, Bakałowicz aurait séjourné cinq mois en Égypte. Le peintre aurait atteint la première cataracte du Nil, aurait visité Thèbes, vu les colosses de Memnon, les pyramides et en aurait fait de nombreux croquis (A. D. 1906, 642-643), qui, s'ils existent encore, doivent être conservés à ce jour dans une collection privée ou dans des réserves de musées.

Le tableau « Le scribe royal Rahotep » réalisé en 1901 (Pl. 1: 1), dont une description a été faite par Dimitri Casali et Caroline Caron-Lefranc de Panthou dans L'Égypte antique par les peintres (2011, 41-43), met en scène un scribe, simplement vêtu d'un pagne et portant une calotte sur la tête, assis en tailleur dos à un mur orné d'une tenture aux motifs géométriques. Le scribe semble réfléchir, les yeux levés, tenant un calame dans la main, prêt à écrire sur le papyrus posé sur une planche qu'il tient sur ses genoux. Quelques plis sur le ventre au niveau du nombril rappellent qu'il a une bonne situation, ce qui n'est pas sans rappeler L'Enseignement de Khéty ${ }^{10}$. Le matériel du scribe, à savoir un vase globulaire ainsi qu'un encrier double, est posé à cté de lui sur un petit meuble. A sa gauche, un second meuble à corniche supporte une bote à couvercle à deux pans au décor bicolore bleu et blanc, ainsi que deux vases : l'un rappelant les vases Nagada, l'autre en albâtre. Au premier plan, les sandales en fibres végétales du scribe sont posées sur une natte. Au-dessus de la tenture, une niche présente une sculpture à l'effigie d'une triade. De part et d'autre de cette niche, une frise court sur le mur, ornée de divinités très facilement reconnaissables : une figure féminine non déterminée suivie des divinités Thot, Isis et Anubis entre lesquelles des cartouches sont inscrits de hiéroglyphes. Thot, le dieu du savoir, inventeur de l'écriture et du langage et donc figure tutélaire des scribes, est reconnaissable à sa tête d'ibis et au matériel de scribe, le calame et la tablette, qu'il tient dans ses mains. Devant lui, Isis, aux bras ailés tenant dans ses mains des clés de vie $\hat{a} k \mathrm{kh}$, porte quant à elle une coiffe constituée de cornes encadrant un disque solaire. A l'autre extrémité, la figure d'Isis

fidèle d'objets par rapport aux autres tableaux réalisés après 1903. Cependant, l'œuvre «La danse égyptienne » réalisée bien plus tôt en 1888 - et qui ne fait pas l'objet de cet article - contredit largement cette pensée en révélant des détails très intéressants confirmant bien la volonté de Bakalowicz de s'attacher à l'introduction de détails crédibles et authentiques dans des compositions qui sont pourtant le fruit de son imagination.

${ }^{10}$ L'Enseignement de Khéty est une satire égyptienne datant de la XII ${ }^{\text {ème }}$ dynastie vantant tous les avantages du métier de scribe en noircissant à l'extrême la condition de tous les autres métiers et dont la principale source textuelle est le papyrus Sallier 2 rédigé en hiératique (XIX ${ }^{\text {ème }}$ dyn. Londres, British Museum, Nr. inv. EA10182).

Helck, W. 1970. Die Lehre des DwA-xtjj. Wiesbaden. 
est répétée en symétrie. Derrière elle, on reconnaît Anubis à tête de chacal tenant une clé de vie $\hat{a} n k h$.

Une triade dite osirienne, figurant de gauche à droite les divinités Horus, Osiris et Isis, prend place dans une niche. Ces trois divinités majeures du panthéon égyptien sont assises. Au centre, Osiris, coiffé de la couronne atef constituée de la couronne de Haute-Égypte flanquée de deux hautes plumes d'autruche, porte de sa main gauche le sceptre nekhakha et de sa main droite le sceptre heqa étonnamment recourbé vers l'extérieur et non vers l'intérieur comme il est conventionnel de le représenter dans la plupart des cas. Il existe de nombreuses triades divines dans l'Égypte ancienne mais celle-ci est la plus répandue (Casali et Caron-Lanfranc de Panthou 2011, 43). Il est difficile de trouver un parallèle archéologique proche de cette œuvre car nombreux sont les détails qui diffèrent de la plupart des triades osiriennes existantes : position assise, pas d'enlacement d'Osiris par Horus et Isis, position des bras croisés d'Osiris, sens des sceptres, forme des perruques, etc. La triade osirienne la plus ressemblante mais qui ne correspond pas entièrement à celle représentée par Bakałowicz est une triade en grauwacke de la $\mathrm{XXVI}^{\text {ème }}$ dynastie conservée dans une collection privée en Floride ${ }^{11}$. Il s'agit d'une triade tenue par une statue naophore malheureusement très fragmentaire.

$\mathrm{Au}$ centre du tableau et assis en tailleur dos au mur, le scribe est une représentation classique dans l'art de l'Égypte ancienne. Levant les yeux vers l'ouverture qui laisse entrer la lumière naturelle éclairant la niche renfermant la triade osirienne, le scribe semble être rêveur. On peut se demander quelle a été la source d'inspiration de Bakałowicz, d'autant plus que ce tableau a été réalisé deux ans avant qu'il se soit rendu en Égypte. Comme le souligne Irina Bogoslovskaya, historienne de l'art russe spécialiste de Stefan Bakałowicz, la source la plus probable semble avoir été « le scribe accroupi » conservé au musée du Louvre à Paris (Богословская И. 2016, 394). On peut supposer que Bakałowicz ait vu lui-même l'œuvre au musée du Louvre (P1. 1:2) ou bien tout simplement dans les planches de l'ouvrage Aegypten in Bild und Wort de Ebers (P1. 1: 3) ou bien dans les Denkmaeler aus Aegypten und Aethiopien de Lepsius (P1. 1: 4) dans lequel se trouve également une représentation de la tête $\mathrm{Salt}^{12} \mathrm{du}$ musée du Louvre dont

\footnotetext{
${ }^{11}$ Triade osirienne, grauwacke, XXVI ${ }^{\mathrm{ème}}$ dyn., H. : 25,4cm, L. : 9,5cm, Pr. : 19cm, collection privée. Royal-Athena Galleries, New York. Extrait de http://www.royalathena.com/PAGES/ EgyptianCatalog/Stone/BM0801C.html (consulté le 13.08.2020).

12 Tête d'homme dite tête Salt, calcaire polychrome, H. : 20,5cm, ancienne collection Salt, Paris, musée du Louvre, Nr inv. N2289.
} 
les traits du scribe de Bakałowicz pourraient se rapprocher (Pl. 1: 5). Cependant, si l'on attire notre attention sur le nom Rahotep donné par Bakałowicz au scribe, cela pourrait nous indiquer qu'il se serait éventuellement inspiré d'une statue d'un dénommé Rahotep. Ce n'est certes pas une statue de scribe, mais la statue de Rahotep et Nofret ${ }^{13}$ conservée au musée égyptien du Caire pourrait en être un modèle très probable. Les lèvres, plus épaisses que celles du " scribe accroupi » du Louvre, ressemblent davantage à celles du scribe de Bakałowicz, bien que l'on puisse noter l'absence de moustache dans sa représentation. Bien que le peintre n'ait pas encore séjourné en Égypte, l'ouvrage Aegypten in Bild und Wort de Ebers publié en $1880(\mathrm{Pl}$. 2: 1-2) en présentait déjà deux illustrations tout comme l'ouvrage Monuments divers recueillis en Égypte et en Nubie de Mariette publié en 1872 (Pl. 2: 3). Par ailleurs, un détail particulier attire l'attention : la calotte crânienne qui coiffe la tête du scribe dans le tableau de Bakałowicz n'est que pure invention, bien que largement répandue dans la représentation que l'on se fait de l'Égypte ancienne, probablement influencée par l'ouvrage The manners and customs of the Ancient Egyptians (Wilkinson 1847, 354, no.1), qui dans une illustration présentant une liste des différentes sortes de perruques, désigne ceci comme un close cap. Il s'agit en réalité de la simple transcription par le dessin d'un crâne rasé et de la marque des cheveux sans perruque, à moins qu'il ne s'agisse d'une confusion ou du moins d'une association d'idées avec la calotte de la divinité égyptienne Ptah.

Un autre détail de ce tableau "Le scribe royal Rahotep » révèle chez Bakałowicz une vision occidentale de l'écriture. Le papyrus tenu par le scribe est en position verticale (en format portrait) alors que celui-ci devrait être présenté à l'horizontale. Les papyri se présentaient en rouleaux et se lisaient à l'horizontale. Les lignes d'écriture sont bien visibles et le scribe semble de surcroît écrire de gauche à droite, sens inverse de l'écriture conventionnelle de l'égyptien ancien, à savoir de droite à gauche.

Dans un deuxième tableau égyptisant de Bakałowicz intitulé "Intérieur de harem égyptien ${ }^{14}$ et réalisé en 1919 (P1. 3: 1), une femme est représentée avec un éventail ${ }^{15}$ à la main dans un intérieur richement décoré. Celle-ci,

\footnotetext{
${ }^{13}$ Statues de Rahotep et Nofret, calcaire polychrome, IV ème dyn., Caire, Musée égyptien, Nr. inv. CG 3 et CG 4.

${ }^{14}$ Également intitulé « Douce intimité » (« Traute Zweisamkeit»), nommé ainsi par la maison de vente Dorotheum.

${ }^{15}$ Le parallèle avec un véritable éventail égyptien antique n'a pas été établi mais il semblerait que les éventails égyptiens soient plus probablement constitués de plumes ou de d'éléments végétaux comme des feuilles.
} 
à la pose mélancolique, est assise sur un lit aux protomes de lions face à une ouverture la baignant d'une lumière chaude. Les décors muraux sont riches en détails et présentent un agencement de nombreux motifs géométriques et de scènes d'adoration de divinités ${ }^{16}$. Il émane de cette scène une atmosphère particulière entre quiétude et ennui profond. Comme le précise le galeriste Jean-François Heim à propos d'un autre tableau de Bakalowicz très similaire à celui-ci : «c'était un joli tableau peint finement présenté dans un cadre néo égyptien probablement d'origine ; Une ouvre d'où se dégageait un climat un peu étrange proche de Gérôme, de Gustave Boulanger et de Lecomte $d u$ Noüy» ${ }^{17}$.

Le motif du lit est particulièrement intéressant puisqu'il présente deux protomes de lions à l'une des extrémités. Ce type de lit n'est pas le fruit de l'imagination de Bakałowicz. Un schéma a été réalisé dans le Catalogue général des antiquités égyptiennes du Musée du Caire de 1914 (P1. 3: 2) dont il aurait pu s'inspirer. Au Museo Egizio de Turin, il existe deux exemplaires de supports en bois pour un lit ornés de lions sculptés de face au museau encadré d'une large crinière stylisée retombant sur la poitrine ( $\mathrm{Pl} .3: 3$ ), très semblables à ceux représentés par Bakałowicz. D'autres protomes de lit en forme de lion sont conservés au British Museum de Londres ${ }^{18}$ ainsi qu'un autre au musée du Louvre ${ }^{19}$ pour lequel il ne s'agit cependant que de deux fragments, seules les têtes ayant été conservées.

Le repose-pieds présent dans ce tableau est un élément de mobilier qui n'a pas nécessairement été considéré comme un élément purement égyptien par Bakałowicz puisqu'il a également utilisé ce motif dans deux de ses autres peintures reconstituant une scène d'intérieur de l'époque romaine : «Femme

\footnotetext{
${ }^{16}$ A mon grand regret, je n'ai à ce jour pas réussi à établir de correspondances entre ces scènes d'adoration et de véritables représentations égyptiennes malgré des recherches dans de nombreuses bases de données de musées, dans ma base de données personnelle ainsi que dans de nombreux ouvrages que Bakalowicz aurait pu consulter à savoir la Description de l'Egypte, les Denkmaeler aus Aegypten und Aethiopien de Lepsius, les Monuments de l'Egypte et de la Nubie de Rossellini, les Planches du Voyage dans la Basse et la Haute Egypte pendant les campagnes du général Bonaparte de Denon, l'Histoire de l'art égyptien d'après les monuments depuis les temps les plus reculés jusqu'à la domination romaine de Prisse d'Avennes ou l'Aegypten in Bild und Wort de Ebers.

${ }^{17}$ Impressions de Jean-François Heim, galeriste ayant présenté le tableau "Princesse égyptienne » dans sa galerie au Louvre des Antiquaires à Paris en 1983, suite à une prise de contact par courriels en mai 2019 au sujet de Bakałowicz.

${ }^{18}$ Fragments de lit égyptien antique avec protomes de lions, bois, époque tardive, H. : 40,4cm, Londres, British Museum, Nr. inv. : EA2522.

${ }^{19}$ Fragments de lit égyptien antique avec protomes de lions, bois, Paris, musée du Louvre, Nr. inv. : E13271, AF 27.
} 
lisant dans un intérieur pompéien » et «Jeune femme lisant 》(P1. 3: 4) réalisé en 1922. Dans le même style d'objet, un repose-tête est intégré à la scène du tableau, objet courant du quotidien qui se retrouve régulièrement dans le mobilier funéraire du défunt dans les tombes, attesté par les nombreux exemplaires présents dans les collections européennes notamment dans celle du musée du Louvre ${ }^{20}$. Celui-ci est de forme très commune et compte parmi les objets très communs dans l'Égypte ancienne. Bakałowicz a su identifier les objets domestiques caractéristiques des intérieurs égyptiens antiques.

Dans ce tableau, on remarque également la tenue vestimentaire et les accessoires portés par la jeune femme représentée relevant davantage d'une interprétation personnelle que d'une reconstitution rigoureuse et scientifique s'appuyant sur des parallèles archéologiques, la robe en étant le parfait exemple. On remarque que celle-ci est une robe à deux bretelles laissant la poitrine nue et dégagée. On peut supposer qu'il s'agit d'une liberté créative prise par Bakałowicz ou d'une erreur de compréhension dans la représentation des robes de l'Égypte ancienne, en raison des représentations en aspective montrant les seins de chaque côté de la robe sur les bas-reliefs et peintures murales antiques. Dans l'ouvrage Pharaonic Egyptian Clothing de Gillian Vogelsang-Eastwood (1993, 105, fig. 7 :7), aucune robe munie de bretelles laissant les seins nus n'est attestée, malgré les multiples variations dans l'apparence des bretelles de robes portées dans l'Égypte ancienne que laisse voir le schéma qu'il propose dans son ouvrage.

Soucieux des détails, Bakałowicz s'est par ailleurs attaché à représenter des bijoux : colliers, boucles d'oreilles, bracelets et broches. La jeune femme porte un collier typiquement égyptien, le collier dit ousekh, signifiant « large ». Ce type de collier, porté par l'élite, est attesté pendant quasiment toute la durée de la civilisation pharaonique, de la $V^{\text {ème }}$ dynastie à 332 av. J.-C. environ. Les boucles d'oreilles portées par la femme assise sur le lit semblent être très fortement inspirées des boucles d'oreilles en or trouvées en juin 1859 par l'égyptologue Auguste Mariette à Abydos en Haute-Égypte et conservées au musée égyptien du Caire (P1. 4: 1). Cellesci sont constituées de plusieurs niveaux de pendeloques dont cinq uraei sur un seul rang (Vernier 1927, 113).

Dans les tableaux de Bakałowicz, aucune débauche d'éléments égyptisants n'est visible. Le peintre ne cède pas à la répétition de motifs inutiles cependant largement exploités par les peintres égyptisants contemporains qui s'inscrivent dans une tradition de répétition des mêmes motifs égyptisants. En effet, au fil des siècles, et ce depuis la Renaissance,

${ }^{20}$ Appui-tête, bois, Paris, musée du Louvre, Nr. inv. : E14440 ou E11989 par exemple. 
une véritable tradition visuelle de représentation de l'Égypte ancienne s'est développée, enrichie de nouveaux motifs au XIX ${ }^{\text {mè }}$ siècle (Moser 2015, $\left.1297^{21}\right)$. Ce « catalogue » de références visuelles ne concentre que des motifs possédant l'essence esthétique et symbolique de l'Égypte ancienne. La puissance évocatrice de ces références iconographiques a incité les peintres à les utiliser comme icônes visuelles de l'Égypte ancienne (Moser 2015, 1297). Les icônes visuelles que sont les pyramides, les obélisques, les sphinx, les momies, les scarabées, les figures d'Antinoüs, les façades de temples, les pharaons, les némès, la figure de Cléopâtre, les yeux oudjat ou encore les sujets bibliques avec Moïse et Ramsès II (l'Exode) sont étonnamment totalement absents des compositions de Bakałowicz. L'absence de ces motifs si évocateurs de l'Égypte ancienne est très étonnante mais révélatrice de la démarche du peintre qui ne cherche pas à transmettre au premier abord une image faussée de l'Égypte par la simple utilisation gratuite de poncifs largement répandus. Toutefois, on remarque une utilisation répétitive de certains motifs discrets tout autant évocateurs de l'Égypte ancienne comme les bouquets de fleurs de lotus, les chats, les disques solaires ailés (behedet), les motifs géométriques ainsi que les dieux à tête animale qui, cependant, sont représentés par Bakałowicz avec justesse et rigueur et sans exagération, procurant davantage une impression de réalisme que d'exagération et de caricature. Ainsi, on observe dans ses œuvres une répétition non seulement des compositions, mais également de certains motifs : «Parfois, Bakałovicz se contente de répéter les sujets préférés des spectateurs, parfois il limite les compositions à l'image d'une figure féminine debout sur fond de draperies ou de murs antiques ${ }^{22}$. Que ce soit dans «Le scribe royal Rahotep » ou dans le tableau «Intérieur de harem égyptien », on remarque la reprise du principe de la source de lumière extérieure à la scène, toujours naturelle mais hors-champ, créant un simple petit carré de luminosité sur une partie du décor, rehaussant ainsi les couleurs et permettant de diriger le regard du spectateur tout en créant des ombres avec le mobilier pour accentuer la profondeur de la scène. D'autres motifs récurrents comme le chat, les bouquets de fleurs de lotus, les éventails ou encore les repose-pieds permettent, soit de meubler la scène, soit de donner une contenance à la figure représentée, comme c'est le cas de l'éventail. Il est d'autant plus intéressant de noter que ces motifs

${ }^{21}$ Tous mes remerciements à Madame Stephanie Moser, Professeur d'archéologie à l'Université de Southampton de m'avoir transmis cet article.

22 « Иногда Бакалович просто повторял ичеликом понравившиеся зрителю сюжеты, иногда ограничивался изображением стоящей женской фигуры на фоне античных драпировок или стен », Golitsyn I. Bakalovich, Stepan Vladislavovich. In The Rodon Foundation. Extrait de http://www.rodon.org/art-071206093316 [consulté le 15.08.2020]. 
ne sont pas seulement réutilisés d'un tableau égyptisant à un autre mais sont récurrents dans l'œuvre de Bakałowicz et se retrouvent dans des tableaux aux sujets bien différents comme le motif de l'éventail dans les tableaux «Sur le balcon » (1908) (P1. 4: 2) ou «La boutique » (date inconnue) et le motif du repose-pieds dans les tableaux «Femme lisant dans un intérieur pompéien » et «Jeune femme lisant» (1922) (Pl. 3: 4).

Plus précisément dans "Le scribe royal Rahotep 》 et "Intérieur de harem égyptien », on observe une réutilisation de motifs communs aux deux tableaux. Concernant le petit mobilier, de petites boîtes permettent de meubler les vides dans les tableaux de Bakałowicz. Dans le premier, une boîte à couvercle à deux pans au décor bicolore bleu et blanc prend place sur un meuble à corniche. Dans le deuxième tableau, une boîte à couvercle à deux pans est posée sur une table semblant porter un décor funéraire, mais la faible résolution de l'image ne permet pas d'identifier clairement le décor. Il existe de très nombreuses boîtes à couvercle à deux pans similaires conservées dans les collections égyptiennes européennes comme par exemple, bien que différent, le coffret de Khâtaouy datant de

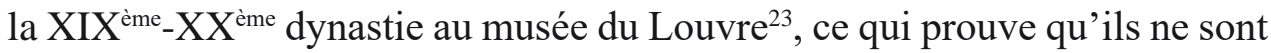
pas le fruit de l'imagination du peintre.

L'attention de Bakałowicz s'est également portée sur les sandales en fibre végétale (papyrus ou palmier). Le peintre en montre de faon très fidèle dans les tableaux "Le scribe royal Rahotep " et "Intérieur de harem égyptien ". L'avant de ces sandales est relevé comme des babouches, celles-ci étant caractérisées soit par la présence, soit par l'absence d'un maintien au niveau du talon. Pour le tableau "Le scribe royal Rahotep ", Bakałowicz s'est inspiré soit de sandales égyptiennes observées dans des collections égyptiennes - puisque ce tableau, contrairement à "Intérieur de harem égyptien », a été réalisé avant son voyage en Égypte en 1903 -, soit de planches de l'ouvrage Recherches sur les arts et métiers, les usages de la vie civile et domestique des anciens peuples de l'Égypte, de la Nubie, et de l'Éthiopie de Cailliaud (P1. 4: 3).

Outre quelques anachronismes spatio-temporels, Bakałowicz prend des libertés dans la représentation et l'introduction de certains motifs. S'inscrivant parfaitement dans l'égyptomanie, Bakałowicz recrée et réinvente une Égypte en réadaptant certains motifs et en introduisant des typologies d'objets non égyptiennes. L'exemple le plus intéressant est la représentation de vases Nagada. Dans les tableaux «Le scribe royal Rahotep » et "Intérieur de harem égyptien », deux vases de type « Nagada » posés sur les meubles

${ }^{23}$ Coffret de Khâtaouy, XIX ${ }^{\text {ème }}-X^{\text {ème }}$ dyn., bois, Paris, musée du Louvre, Nr. inv. N2918. 
sont représentés mais n'en sont pas pour autant, leur décor étant trompeur. Les triangles dans le registre supérieur du vase au niveau de l'épaule (Pl. $3: 1$ ) sont des décors que l'on peut trouver sur les vases Nagada comme par exemple celui conservé au musée du Louvre (P1. 4: 4). En outre, la forme du vase dans le tableau «Le scribe royal Rahotep » ne correspond pas à une typologie de vase égyptien, d'autant plus qu'il n'y a pas de lignes ondulées continues, ni ces autres formes géométriques représentées dans les véritables décors nagadiens. Il est donc peu probable qu'il s'agisse de copies de vases réels. Par ailleurs, ce vase pourrait tout simplement être un des motifs récurrents que Bakałowicz réutilise d'œuvre en œuvre. Dans son tableau «Pax vobiscum » réalisé en 1900 (Pl. 5: 1), on peut remarquer, dans une niche à l'arrière-plan, un vase ayant exactement la même typologie de vase mais n'ayant évidemment pas les décors prétendument nagadiens. Concernant les autres vases représentés, le vase globulaire sur la petite table pourrait s'inspirer d'un vase datant de l'Ancien Empire, mais ce de forme trop " simple » pour en trouver un modèle exact éventuel. Le vase en albâtre, à côté du vase décoré, pourrait bien s'inspirer de vases égyptiens en pierre datant davantage de l'époque tardive mais l'absence de lèvre sur celui-ci ne permet pas d'établir un parallèle avec un véritable vase égyptien antique qui devrait en présenter une dans les typologies s'en rapprochant. La forme du vase double, ayant très certainement la fonction d'encrier, n'est pas habituel non plus et aucun parallèle archéologique ne semble confirmer qu'il s'agisse d'une reproduction d'un objet égyptien. Par ailleurs, le vase bleu avec des fleurs dans le tableau «Intérieur de harem égyptien » n'est de toute évidence pas égyptien mais daterait du XIX ${ }^{\text {ème }}$ ou du $\mathrm{XX}^{\mathrm{ème}}$ siècle $^{24}$. Ces reconstitutions hypothétiques ne sont pas pour autant à blâmer. Le rôle de l'égyptomanie n'est pas de proposer une reconstitution scientifique mais de faire revivre un passé oublié.

En raison de ses multiples sources d'inspiration, qu'il s'agisse de quelques ouvrages égyptologiques, d'ouvrages de compilation de motifs et d'œuvres égyptiennes, d'objets conservés dans les collections égyptiennes ou par ceux qu'il a pu esquisser lors de son propre séjour en 1903 en Égypte, Bakałowicz fait preuve d'une précision archéologique très étonnante pour retranscrire au mieux l'atmosphère de l'Égypte ancienne et réussir à plonger le spectateur de ses œuvres dans une Égypte récréée et ressuscitée par un dessin fidèle, malgré quelques libertés prises. Mais comme nous l'avons

\footnotetext{
${ }^{24}$ Tous mes remerciements à Monsieur Stan Hendrickx, égyptologue belge, pour son avis éclairé au sujet des décors et typologies des vases Nagada, relatif aux vases représentés par Bakałowicz.
} 
évoqué précédemment, la représentation de l'Égypte au XIX ${ }^{\text {ème }}$ siècle ne doit pas nécessairement être considérée uniquement à travers le prisme de la tradition égyptomaniaque mais également de celui de l'orientalisme. Le harem, véritable leitmotiv de la peinture orientaliste, thème très en vogue au $\mathrm{XIX}^{\mathrm{ème}}$ siècle, est le sujet du tableau "Intérieur de harem égyptien » dans lequel s'exprime par ailleurs le thème de la mélancolie. On ne sait si la femme au bord du lit exprime la paresse, la solitude, l'ennui, la tristesse, l'affliction, la peine, l'abattement ou encore la méditation. Elle a le regard perdu dans le lointain. Tous ces éléments peuvent être regroupés sous la notion de «mélancolie », dont la femme adopte en outre la pose classique et très représentative : la tête inclinée vers le sol s'appuyant presque sur la main (Demont P., La mélancolie dans l'Antiquité : de la maladie au tempérament. In Clair 2005, 34). Cette représentation canonique de la mélancolie trouve déjà son origine dans l'Antiquité comme par exemple sur le décor à figures rouges d'un skyphos conservé à Chiusi au Museo Archeologico Nazionale ${ }^{25}$ ou sur le bronze d'Ajax ${ }^{26}$. Ces deux derniers exemples cités permettent seulement d'illustrer le fait que cette représentation était déjà présente durant l'Antiquité mais n'ont pas servi pour autant de modèles à Bakałowicz. Dans son Iconologia overo Descrittione dell'Imagini universali, l'érudit italien Cesare Ripa (1555-1622) écrit en 1593 au sujet de la figure de la mélancolie : «Femme âgée, triste et dolente, habillée de méchants vêtements, sans ornement aucun, assise sur un rocher, les coudes posés sur les genoux avec, près d'elle, un arbre dépouillé, et des rochers épars ${ }^{27}$. Cette représentation classique de la mélancolie s'est répandue tout au long du XVII ${ }^{{ }^{2} m e}$ siècle et a été adaptée ici au goût de l'époque et au sujet exotique. On peut donc justement s'interroger sur la fonction des personnages représentés dans les tableaux de Bakałowicz. Sont-ils des figures vivantes ou des figures meublant l'espace ? Font-ils partie du décor ou permettent-ils de donner

\footnotetext{
${ }^{25}$ Représentation de Pénélope dans une affliction profonde devant son métier à tisser, sa tête voilée et inclinée est soutenue par sa main droite. Skyphos à figures rouges: Télémaque et Pénélope devant son métier à tisser, style attique, vers 440-435 av. J.-C., argile, 20,5 x 24,5cm, attribué au Peintre de Pénélope (vase éponyme), Chiusi, Musée archéologique national, Nr. inv. 62705. Reproduit dans Ortiz G. 2005. Ajax. In Clair 2005, 49, fig. 2.

${ }^{26}$ Ajax, bronze, H.: c. $29 \mathrm{~cm}$, L. max.: c. $33 \mathrm{~cm}$, Asie Mineure, romain, début de l'époque d'Auguste.

Reproduit dans Ortiz G. 2005. Ajax. In Clair 2005, 51, fig. 6.

${ }^{27}$ Planche Malinconia de Cesare Ripa extraite de Della Novissima Iconologia, Padoue, 1624-1626, gravure sur bois, 22,8 x 15,4cm, Paris, Bibliothèque nationale de France. D'après Mélancolie. Génie et folie en Occident. In Petit journal des grandes expositions. Paris. 383, 7.
} 
vie au décor ? Cependant, sont-ils des personnages mis en scène au milieu d'un décor égyptisant ou est-ce justement un décor à l'égyptienne rendu vraisemblable et plausible par l'introduction de personnages ? Bien qu'il n'y ait aucun dynamisme particulier à proprement parler, on peut considérer que les tableaux sont de véritables tableaux vivants dans le sens où les figures sont mises en scène par leur placement dans la scène et leur mise en valeur par la source de lumière extérieure au tableau. Le spectateur peut s'identifier à ces personnages, ce qui renforce le caractère immersif de l'œuvre.

Un autre élément purement oriental est présent dans le tableau « Le scribe royal Rahotep ». Un motif en frise de pyramide à degrés est représenté sur la tenture sur laquelle s'appuie le scribe assis en tailleur. Ce motif n'est pas caractéristique de l'Égypte ancienne. Un dernier ouvrage non égyptologique mais particulièrement important est la Grammar of Ornament de Owen Jones dans lequel de nombreux artistes à la recherche de motifs ont puisé leur inspiration au XIX ${ }^{\text {ème }}$ siècle. On retrouve donc ce motif sur les planches 32 (Pl. 5: 3) et 33 de cet ouvrage (Owen 1868, Tafel XXXII, no. 1-7) qui a très certainement été la source d'inspiration de Bakałowicz. Ce motif est par ailleurs présent dans un tableau orientaliste contemporain, « Le Charmeur de serpents » de Francesco Coleman (P1. 5: 2), dans lequel il est représenté de la même manière, en frise. Cet élément décoratif est emprunté à un élément architectural oriental qu'est le couronnement en merlons à degrés caractéristique des terrasses horizontales. Ce type de couronnement se retrouve déjà à la fin du troisième millénaire av. J.-C. jusqu'aux villes d'Ur (ziggurat) et d'Uruk (temple d'Innina) en Mésopotamie. Un des tableaux du peintre contemporain Lecomte du Noüy (1842-1923) «Le Songe de l'eunuque » (P1. 5: 4) réalisé en 1874 et conservé au Cleveland Museum of Art montre ce motif architectural. Comme le précise Adnan Bounni (1999, 508), deux sortes de merlons existaient dans la civilisation égyptienne, l'un en forme de gerbe de papyrus (Pl. 6: 1), l'autre en forme de demi-cercles (Pl. 6: 2), qui diffèrent cependant en forme et en fonction de ceux d'Orient. Les murs, notamment des temples, étaient en Egypte ordinairement couronnés de corniche à gorge. Cette introduction d'un motif purement oriental dans une composition ayant pour sujet l'Égypte ancienne est caractéristique de la vision romantique de l'orientalisme qui associe l'Orient contemporain et l'Orient antique selon une idée d'immuabilité et d'intemporalité face à un monde européen en pleine mutation (Thornton 1983, 11).

$\mathrm{Au}$ vu de ce mélange de motifs purement égyptiens, de certains issus du vocabulaire de l'orientalisme et d'autres relevant de la pure fantaisie de Bakałowicz, il est difficile de trancher : est-ce le plaisir scientifique ou bien 
le plaisir esthétique qui prime ? Bakałowicz souhaitait-il instruire ou bien ravir à travers ses tableaux égyptisants, comme selon l'expression allemande « erst belehren oder erfreuen »? Bien que les reproductions d'objets archéologiques soient très précises et que les œuvres soient empreintes d'un réalisme sympathique, plusieurs détails sont pittoresques et fantaisistes. Une impression de plénitude, de temps figé, de sérénité, de calme et de confort s'en dégage, appuyée par un contraste important entre les personnages inactifs et le décor chargé de détails et de couleurs vives et chaudes. C'est une véritable évocation d'une Égypte intemporelle et immuable.

La rigueur de Bakałowicz dans la construction de ses tableaux lui a permis de proposer une image d'une Égypte ancienne ressuscitée - certes dans une certaine mesure fantasmée et rêvée - mais surtout crédible et authentique sans pour autant être exacte. Peintre polonais de première importance et un des derniers représentants de l'académisme tardif, Stefan Bakałowicz a su développer son propre style et répondre au goût bourgeois de l'époque. En cette période d'exaltation, de fascination et de passion pour la civilisation pharaonique qu'est le XIX ${ }^{\text {ème }}$ siècle, il est comparé à son compatriote Henry Siemiradzki et le célèbre " peintre-archéologue » Lawrence AlmaTadema, parangon de cette peinture historiciste de cette seconde moitié du XIX ${ }^{\text {ème }}$ siècle. Entre peinture d'histoire et peinture de genre, les tableaux de Bakałowicz sont des images plaisantes à regarder, proposant une vision séduisante d'une Égypte ancienne disparue teintée d'un orientalisme et d'un exotisme charmants. Entre reconstitution, restitution et imaginaire, la plénitude qui enveloppe ses œuvres incite à la rêverie. 


\section{References}

A. D. 1906. Stefan Bakałowicz. In Tygodnik Illustrowany 33, Varsovie, 642643.

Benezit E. Dictionnaire des Peintres, Sculpteurs, Dessinateurs et Graveurs 1. Paris.

Богословская И. 2016. Малоизвестные страницы жизни и творчества С. Бакаловича по материалам российской и польской прессы. In Malinowski J. et Gavrash I. (eds). Генрих Семирадский и академизм, Искусство Востоуной Европьы. Warszawa-Toruń, 385-399.

Bounni A. 1999. Couronnement des sanctuaires du Proche-Orient hellénistique et romain : origine et développement du merlon. In: Topoi 9/2, 507-525.

Boyer V. 2019. Die ägyptisierenden Gemälde des russisch-polnischen Malers Stefan Bakałowicz (1857-1947). Zwischen Ägyptomanie und Ägyptophilie: Inspirationen, Einflüsse sowie künstlerische und ästhetische Ansätze eines Malers des Spätakademismus. Unpublished MA dissertation, University of Heidelberg. Heidelberg.

Cailliaud Fr. 1831. Recherches sur les arts et métiers, les usages de la vie civile et domestique des anciens peuples de l'Égypte, de la Nubie, et de l'Éthiopie. Suivies de détails sur les mours et coutumes des peuples modernes des mêmes contrées. Paris.

Casali D. and Caron-Lanfranc de Panthou C. 2011. L'Égypte antique par les peintres. Paris.

Clair J. (ed.) 2005. Mélancolie, génie et folie en Occident, catalogue d'exposition Grand-Palais Paris du 10 octobre 2005 au 16 janvier 2006. Paris.

Derwojed J. 1971. Stefan Bakałowicz. In J. Maurin-Bialostocka (ed.). Stownik artystow polskich $i$ obcych $w$ Polsce działajacych. Malarze, rzeźbiarze, graficy 1. Wrocław, 72-73.

Ebers G. 1879a. Aegypten in Bild und Wort. Dargestellt von unseren ersten Künstlern, beschrieben von Georg Ebers 1. Stuttgart, Leipzig.

Ebers G. 1879b. Aegypten in Bild und Wort. Dargestellt von unseren ersten Künstlern, beschrieben von Georg Ebers 2. Stuttgart, Leipzig.

Günther R. 1914. Catalogue général des antiquités égyptiennes du Musée $d u$ Caire. Naos. N. 70001-70050. Leipzig.

Hennebert D. (ed.) 2012. Egyptomanies depuis le XIX ${ }^{e}$ siècle. Edouard et Cléopâtre, catalogue d'exposition Fondation Boghossian - Villa Empain du 20 septembre 2012 au 10 février 2013. Brussels. 
Lepsius K. R. 1849-1859. Denkmaeler aus Aegypten und Aethiopien, Abteilung 3, Band 1. Berlin.

Mariette A. 1872. Monuments divers recueillis en Egypte et en Nubie. Paris.

Musée égyptien de Turin 1987. Civilisations des Égyptiens. La vie quotidienne. Milan, 156, fig. 210.

Moser S. 2015. Reconstructing ancient worlds: Reception studies, archaeological representation and the interpretation of ancient Egypt, in Journal of Archaeological Method and Theory 22 (N. 4), 1263-1308.

Owen J. 1868. Grammatik der Ornamente. Illustrirt mit Mustern von den verschiedenen Stylarten der Ornamente in Hundert und zwölf Tafeln. London.

Ripa C. 1593. Iconologia overo descrittione dell'imagini universali cavate dall'antichita et da altri luoghi ([Reprod.]) da Casare Ripa Perugino. Roma.

Thornton L. 1985. La femme dans la peinture orientaliste. Courbevoie.

Vernier E. 1927. Catalogue général des antiquités égyptiennes du Musée $d u$ Caire. Bijoux et orfèvreries 2, N. 52001-53855. Caire.

Vogelsang-Eastwood G. 1993. Pharaonic Egyptian Clothing. Leiden.

Wilkinson G. (Sir) 1847. The manners and customs of the Ancient Egyptians, including their private live, government, laws, arts, manufactures, religion, agriculture, and early history 3. London.

Valentin Boyer

Chargé des programmes franco-allemands de soutien à la recherche Centre interdisciplinaire d'études et de recherches sur l'Allemagne valentinjoseph.boyer@gmail.com 


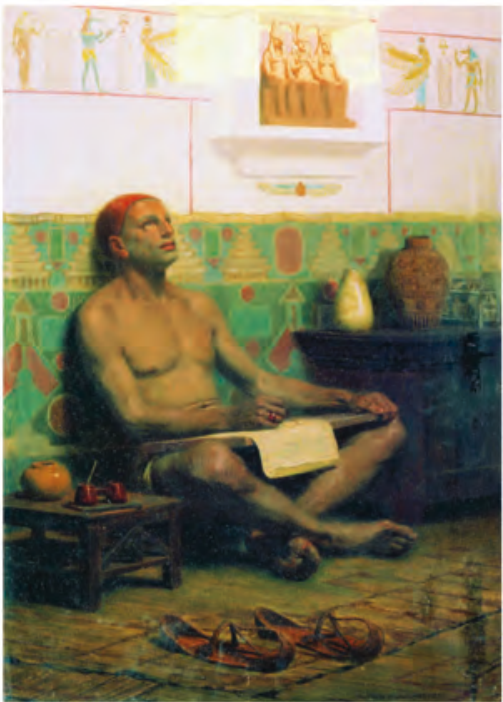

1

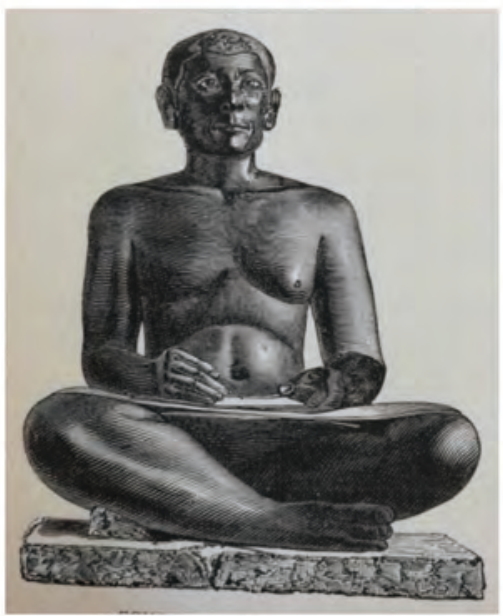

4
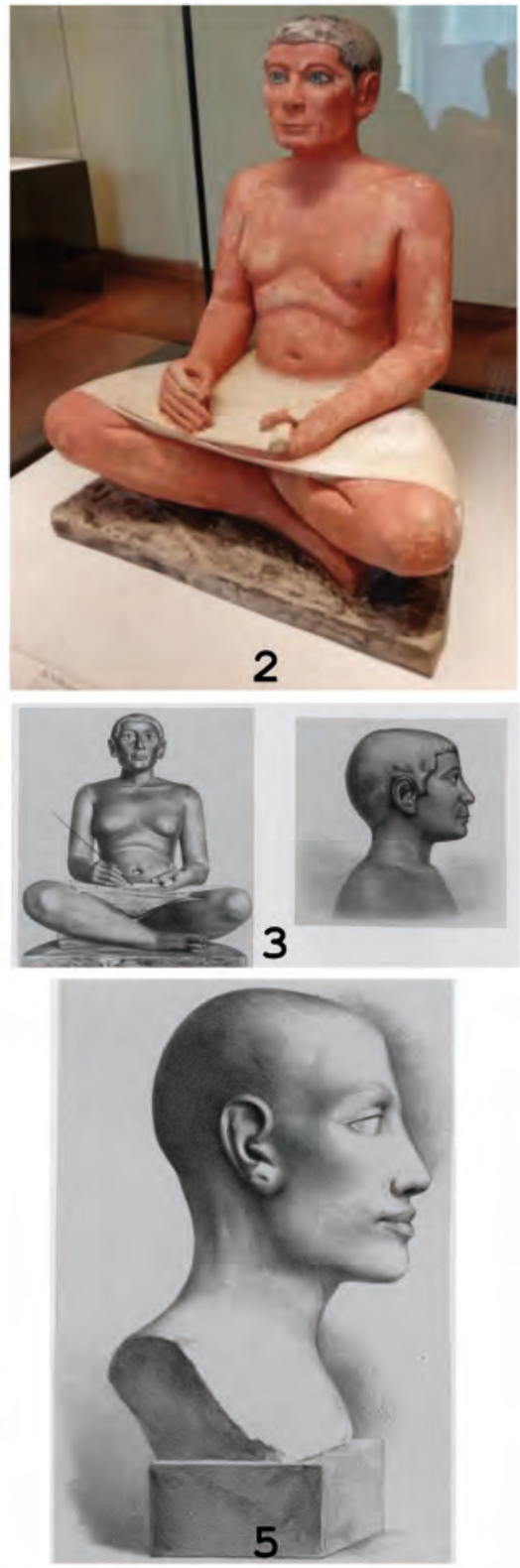

Pl. 1: 1. Stefan Bakałowicz, Le scribe royal Rahotep, huile sur toile, 48 x 33cm, 1901, Yaroslavl, Musée d'art provincial d'tat (photographie du musée d'art provincial d'tat de Yaroslavl)

P1. 1: 2. Le "Scribe accroupi », IV ème/V ${ }^{\text {ème }}$ dynastie (2600-2350 v. Chr.), calcaire polychrome, yeux incrustés de cristal de roche dans du cuivre, H. : $53,70 \mathrm{~cm}, \mathrm{~L}$. : $44 \mathrm{~cm}$, Pr. : $35 \mathrm{~cm}$, Paris, musée du Louvre, Nr. inv. E 3023 (photographie de l'auteur) P1. 1: 3. Le « Scribe accroupi », illustration de B. Strafsberger (d'après Ebers 1879a, 153) Pl. 1: 4. Le "Scribe accroupi » (d'après Lepsius 1849-1859, Abteilung III, Band. 1, Tfl. 290, ouvrage digitalisé par la bibliothèque universitaire de Heidelberg)

P1. 1: 5. Tte d'homme dite tte Salt (d'après Lepsius 1849-1859, Abteilung III, Band. 1, Tfl. 290, ouvrage digitalisé par la bibliothèque universitaire de Heidelberg) 


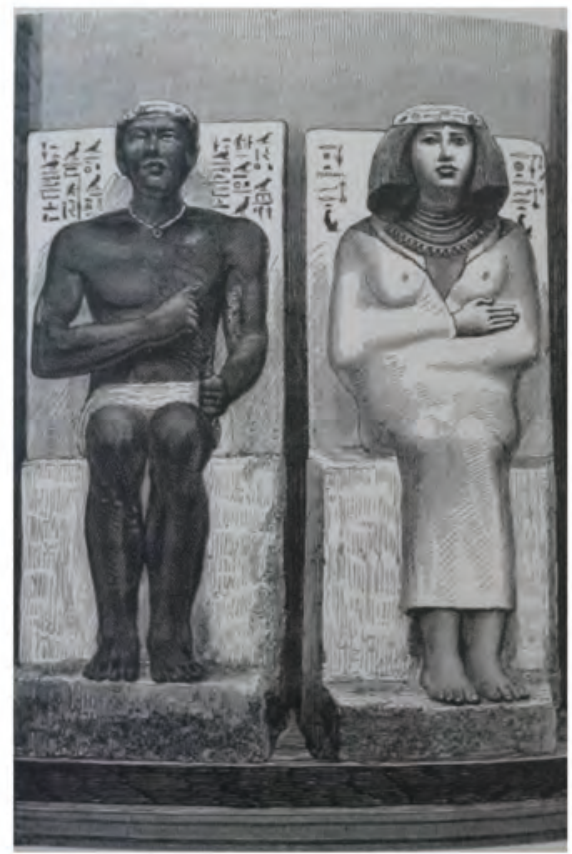

1

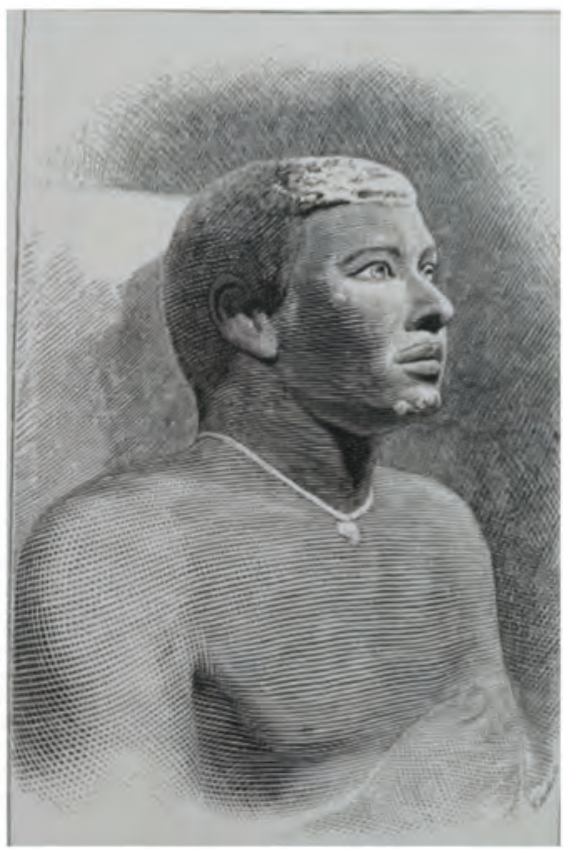

2
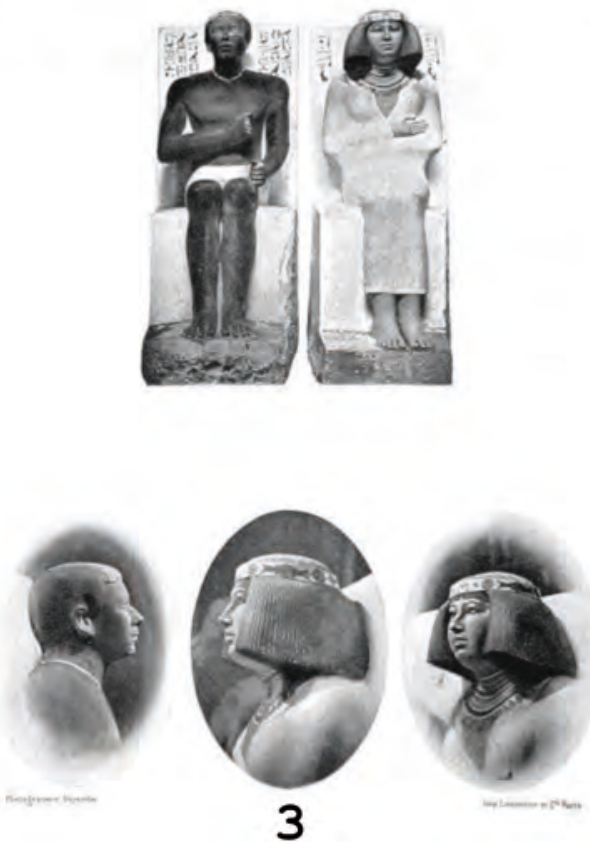

Pl. 2: 1. Rahotep et Nofret, illustration d'August Ramsthal (d'après Ebers 1879b, 52)

P1. 2: 2. Rahotep, illustration d'August Ramsthal (d'après Ebers 1879b, 59)

Pl. 2: 3. Rahotep et Nofret, Meïdoum, tombe n² (d'après Mariette 1872, pl. 20) 


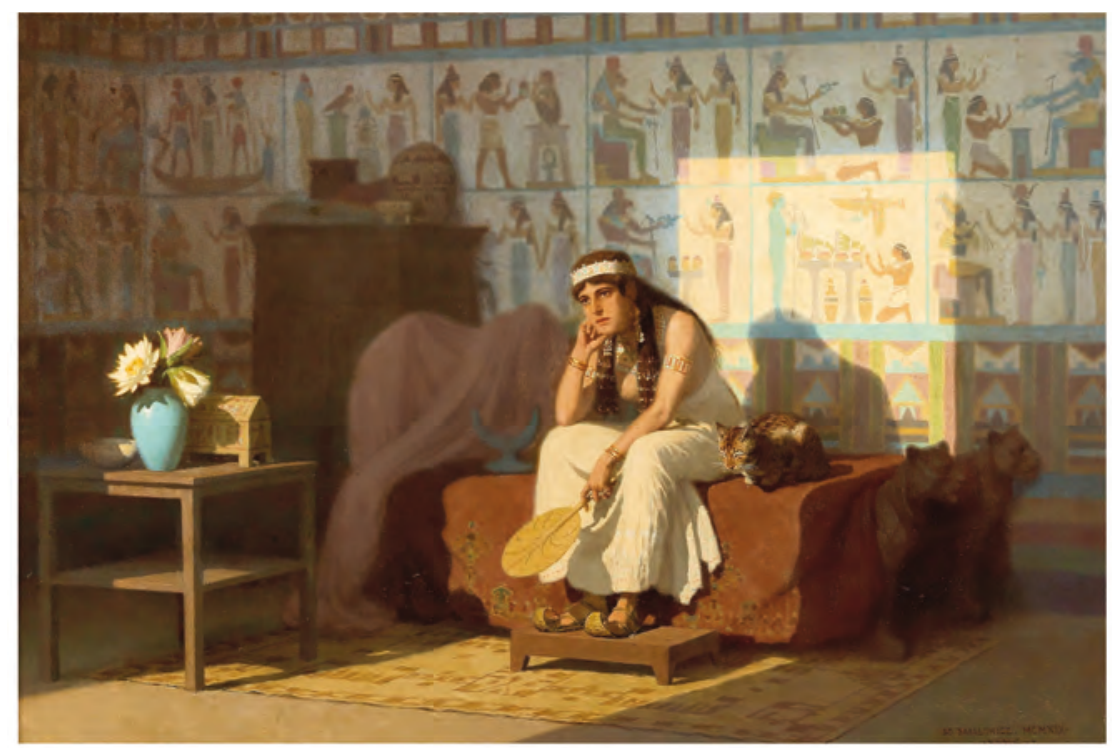

1

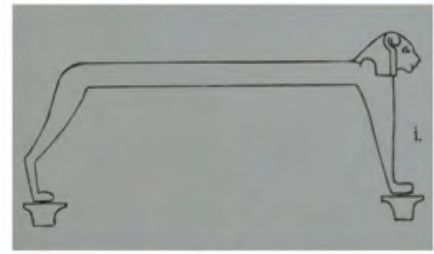

2

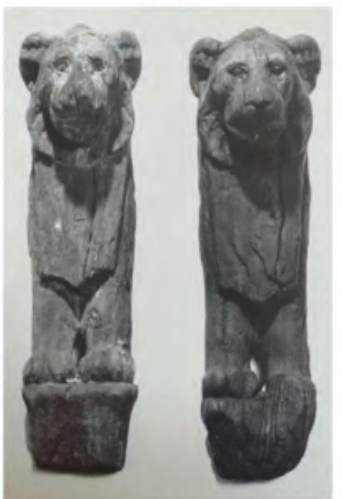

3

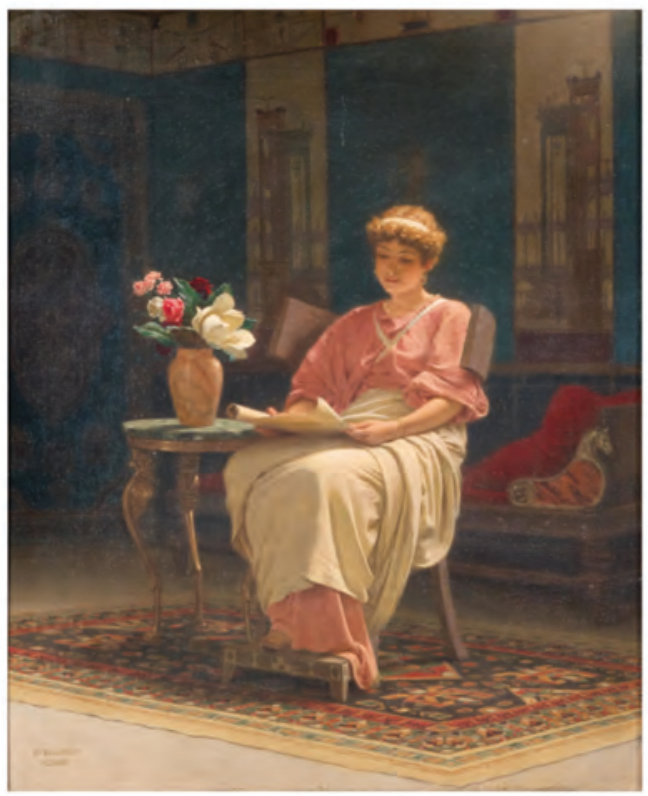

4

P1. 3: 1. Stefan Bakałowicz, Intérieur de harem égyptien, 1919, huile sur bois, 33 x $47.5 \mathrm{~cm}$, signé et daté « St. Bakalowicz MCMXIX, Roma », collection privée (photographie de Dorotheum Wien, Auktionskatalog 19.06.2018)

P1. 3: 2. Schéma de lit égyptien antique (d'après Günther 1914, P1. LXXXII, fig. 1)

P1. 3: 3. Fragments de lit égyptien, Nouvel Empire, bois, H. : 43cm, ancienne collection Drovetti, Turin, musée égyptien, Nr. inv. 6402 (d'après Musée égyptien de Turin 1987, p. 156, fig. 210)

P1. 3: 4. Stefan Bakałowicz, Jeune femme lisant, 1922, huile sur toile, 47 x $38 \mathrm{~cm}$, signé et daté « St. Bakalowicz MCMXXII », collection privée (photographie de Dorotheum Wien, Auktionskatalog 10.12.2018 / domaine public) 


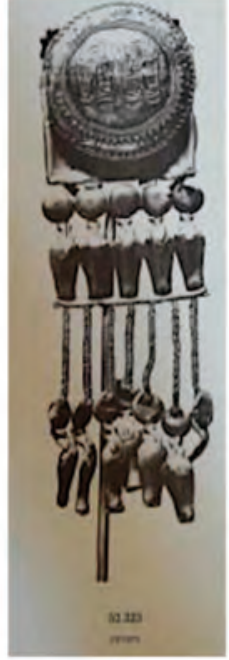

1

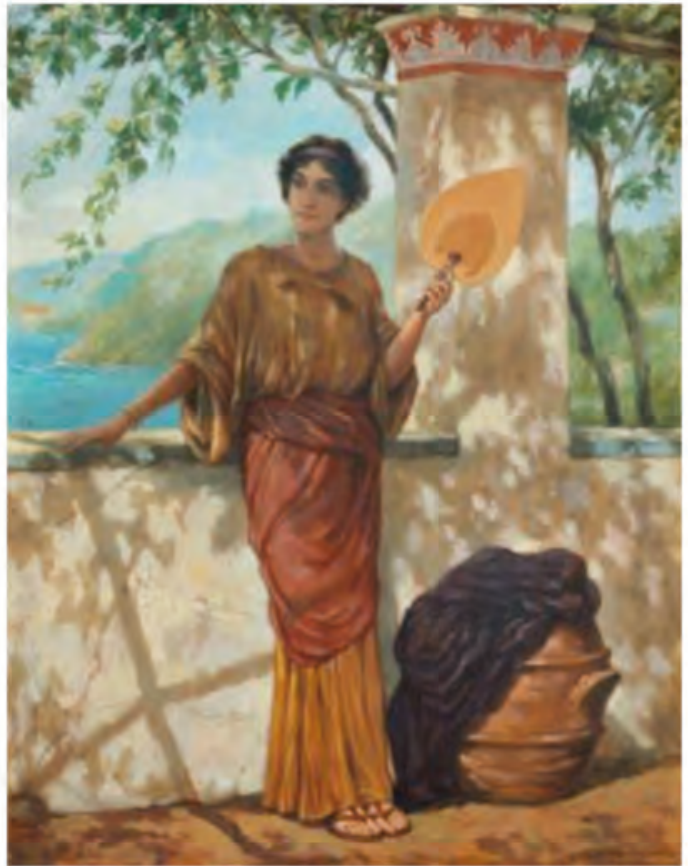

2

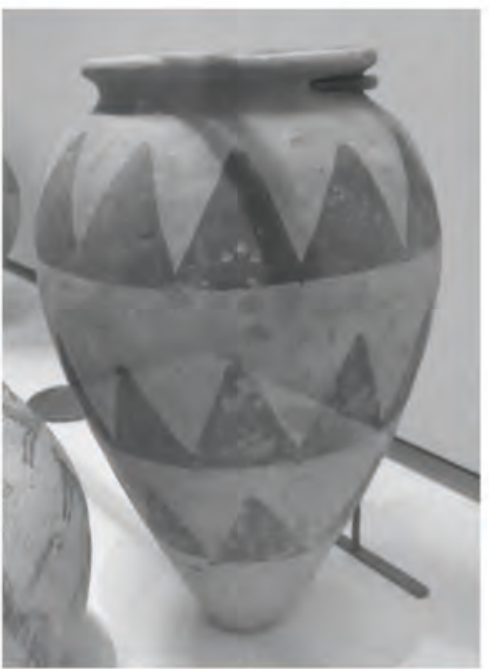

4

Pl. 4: 1. Boucles d'oreilles de Ramsès XII, or, $X^{\text {ème }}$ dynastie, trouvées à Abydos par Mariette en juin 1859, Caire, musée égyptien, Nr. inv. 52.323 (d'après Vernier 1927, Pl. XXVII)

Pl. 4: 2. Stefan Bakałowicz, Sur le balcon, 1908, huile sur toile, collection privée (photographie de Bonhams 2001-2019)

Pl. 4: 3. Schéma de sandales égyptiennes antiques (d'après Cailliaud 1831, Pl. 21) Pl. 4: 4. Vase Nagada, Paris, musée du Louvre, Nr. inv. E 24732 (photographie de l'auteur) 


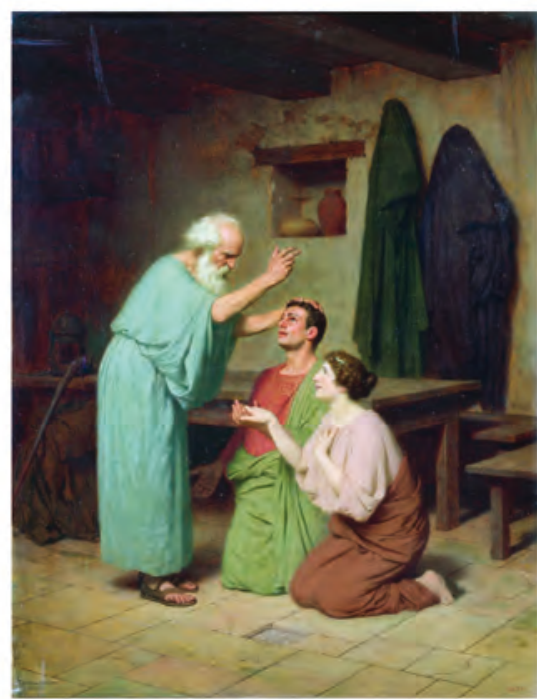

1

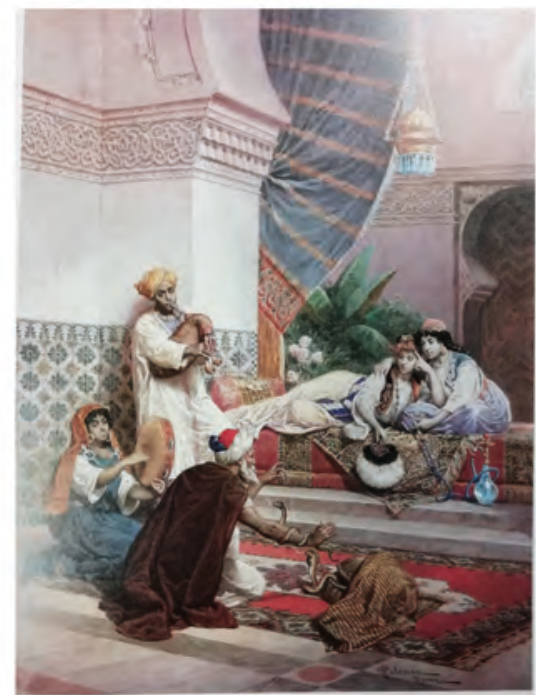

2

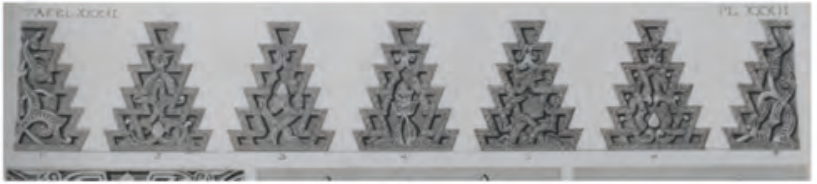

3

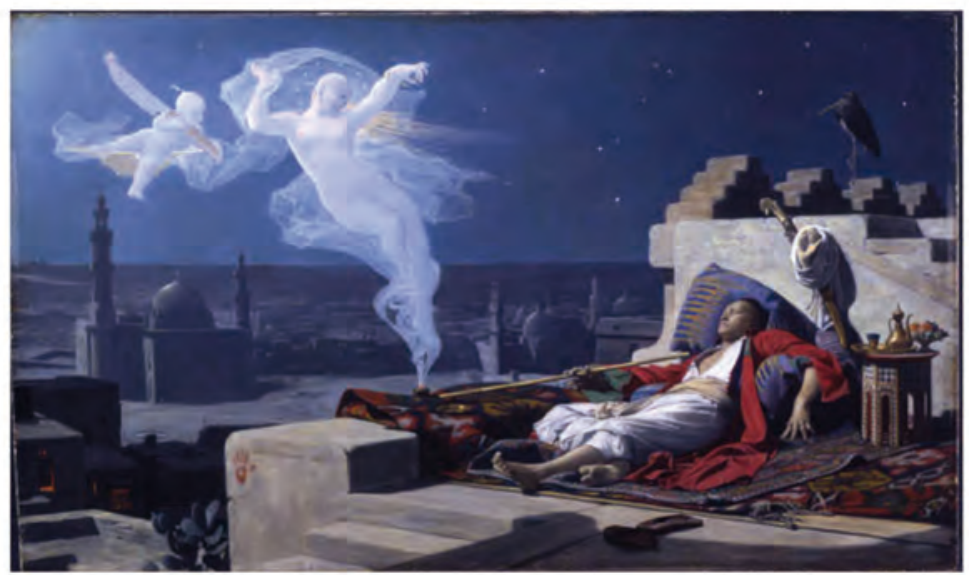

4

P1. 5: 1. Stefan Bakałowicz, Pax Vobiscum, 1900, huile sur toile, 58,5 x 45cm, Tver,

Galerie d'art régional (photographie de 2000-2018 The Athenaeum/ domaine public)

P1. 5: 2. Francesco Coleman, Le Charmeur de serpents, aquarelle, signée, 64 x $47 \mathrm{~cm}$, Anc. Mathaf Gallery, Londres (d'après Thornton 1985, 44)

P1. 5: 3. Motif de merlons à degrés à décor floral (d'après Owen 1868, Tafel XXXII, no. 1-7)

P1. 5: 4. Jean Lecomte du Noüy, Le Songe de l'eunuque, 1874, huile sur toile, $39,3 \mathrm{~cm} \mathrm{x}$ $65,4 \mathrm{~cm}$, Cleveland Museum of Art (photographie de Paulo Cunha Porto / domaine public) 

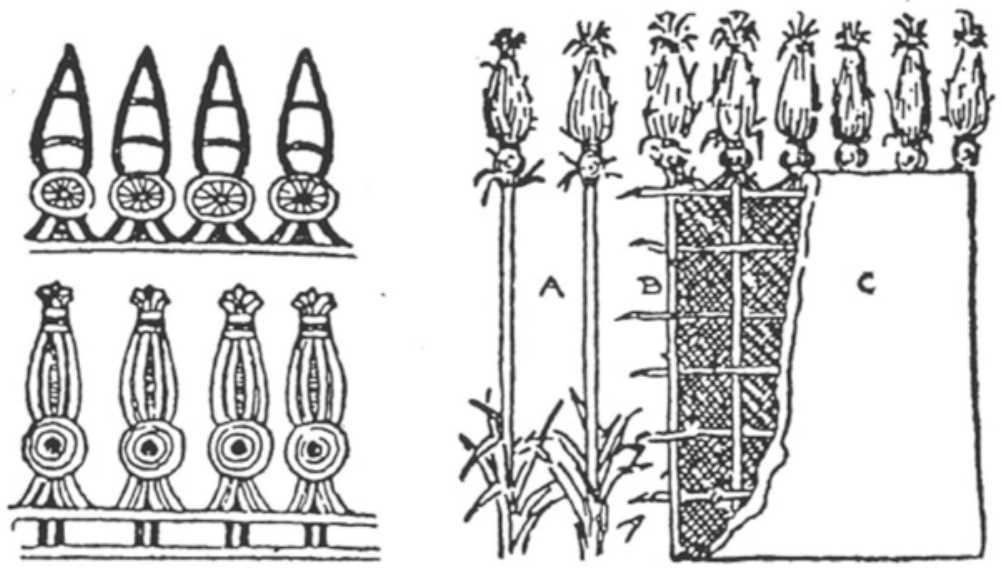

1

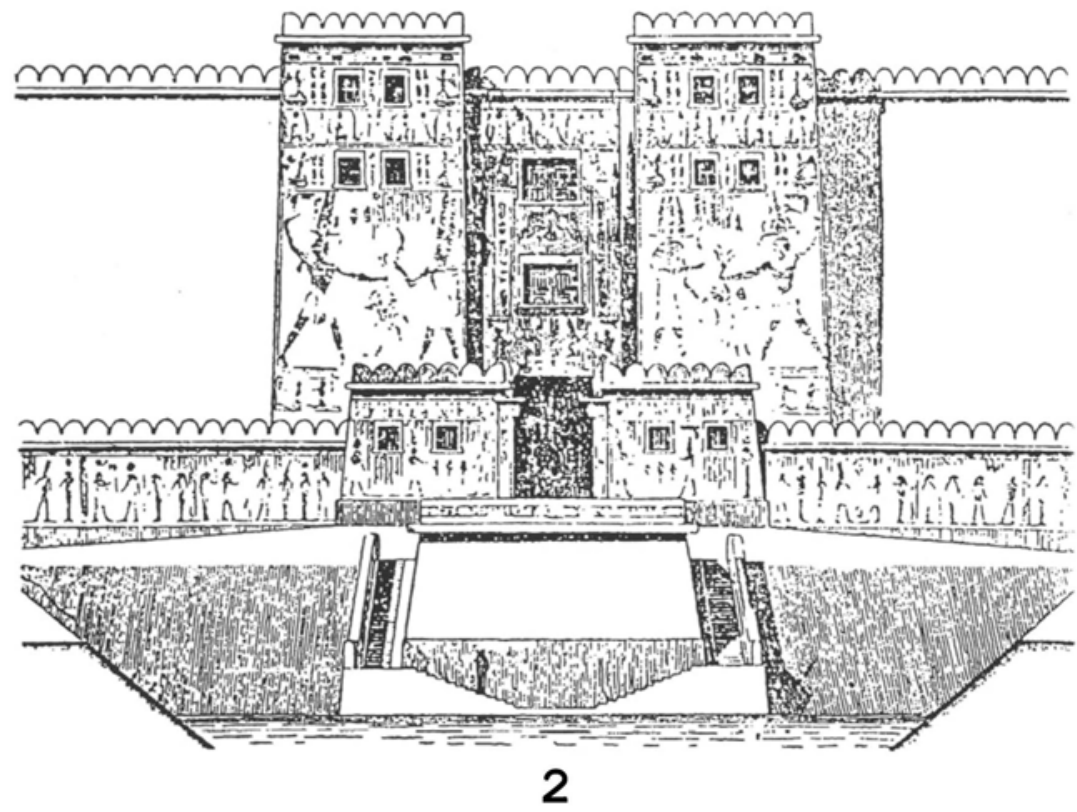

Pl. 6: 1. Merlons en forme de gerbes de papyrus (d'après Bounni 1999, 515, Fig. 3)

P1. 6: 2. Merlons en forme de demi-cercles, remparts de Medinet Habu (d'après Bounni 1999, 515, Fig. 4) 